\title{
Prognostic features of NRAS missense mutations in adult acute myeloid leukemia patients
}

\author{
S.V. Sazonov ${ }^{2,3}, A . V$. Vinogradov $^{1,2}, D . V$. Litvinova $^{2}, A . N$. Loboda $^{2}$, and A.V. Rezaykin ${ }^{2}$ \\ ${ }^{1}$ Sverdlovsk Regional Clinical Hospital N 1,620102, Volgogradskaya Str., 185, Ekaterinburg, Russia \\ ${ }^{2}$ Ural State Medical University, 620028, Repina Str., 3, Ekaterinburg, Russia \\ ${ }^{3}$ Institute of Medical Cell Technology, 620026, Karl Marks Str., 22A, Ekaterinburg, Russia
}

\begin{abstract}
The aim of the study was to assess the prognostic significance of missense mutations in the NRAS gene in adult patients with acute myeloid leukemia (AML). Clinical observation was performed on 70 patients with AML. The average age of the examined was $52.0 \pm 3.4$ years. NRAS gene point mutations were detected using direct sequencing technique.

According to the results of cytogenetic, immunohistochemical and PCR studies, a favorable prognosis was determined in 18 cases $(25.7 \%)$, an intermediate in $15(21.4 \%)$, and an unfavorable one in 18 cases $(25.7 \%)$. In 19 samples (27.1\%) genetic anomalies could not be detected; accordingly, the prognosis option for such patients was not specified.

NRAS missense mutations were represented by T17A, C181A, A182C transversions ( 5 cases) and G35A, G38A transitions (3 cases), and a synonymous (silent) G360A substitution (1 case). Average frequency of prognostically significant NRAS mutations was $11.4 \%$. All the above nonsynonymous mutations were localized in exons $1-3$, which caused the blocking of the internal GTPase activity of the N-ras protein and its stabilization in the active state. Clinically, all NRAS-positive AMLs were characterized by an unfavorable prognosis and primary tumor resistance to chemotherapy. The average follow-up of patients was $8.4 \pm 8.2$ months.
\end{abstract}

\section{Introduction}

Acute myeloid leukemia (AML) is a clonal neoplastic disease of the hematopoietic tissue associated with a mutation in the hematopoietic progenitor cell, which results in differentiation block and uncontrolled proliferation of immature myeloid cells. In addition to standard analyzes for verification of the diagnosis, the European clinical guidelines for the treatment of AML also offer an analysis of bone marrow aspirate for the presence of mutations in certain genes, such as NRAS, ASXL1, FLT3, NPM1, TP53 and others [1].

The NRAS gene is located on the short arm of the first chromosome at position 13.2 and belongs to the RAS proto-oncogen family, which also includes two other genes: HRAS and KRAS. Proteins co-labeled with the indicated genes are small G-proteins that act as 
GTPase molecular signal switches from activated cytoplasmic membrane receptors. In an activated GTP-bound state, they activate a series of downstream effector cascades that ultimately lead to the activation of nuclear transcription factors. The three main effector pathways that are activated by N-ras proteins: NRAF / MEK / MAPK, PI3K / AKT, and RAL-GDS mediate cell proliferation, survival, adhesion, and motility.

NRAS consists of a main G-domain with catalytic functions and a hypervariable region with a lipidized C-terminus with low homology between isoforms, which determines the localization of proteins in specific microdomains. The G-domain of NRAS consists of a 6element $\beta$-sheet surrounded by $5 \alpha$-helices and 10 connecting loops with two switches, switches I and II, which have different conformations in GDP and GTP bound states [2] . The most significant differences are localized in two regions: residues 30 through 38 (switch region I) in the second loop and residues 60 through 76 (switch region II), consisting of the fourth loop and a short alpha helix that follows the loop [3].

NRAS mutations are oncogenic somatic mutations, typical of which cause amino acid substitutions at positions Gly12, Gly13 or Gln61, block N-ras protein in the active state associated with GTP, thereby reducing the internal activity of GTPase. According to [2-5], heterozygous NRAS mutations are detected in $14-17 \%$ of cases of human malignant tumors.

The aim of the study was to assess the prognostic significance of missense mutations in the NRAS gene in adult patients with acute myeloid leukemia.

\section{Materials and methods}

Clinical observation was carried out on 70 patients with AML based on the Sverdlovsk Regional Clinical Hospital No. 1 (Yekaterinburg), of which 33 were men and 37 women. The average age of the examined was $52.0 \pm 3.4$ years.

Morphologically, the diagnosis of AML in all cases was previously verified in specialized laboratories on the basis of the Sverdlovsk Regional Clinical Hospital No. 1 and the Sverdlovsk Regional Pathological Anatomical Bureau using standard cytological, cytochemical, immunophenotypic, histological, and immunohistochemical methods. The study included the following variants of AML according to the WHO classification: M0 - 4, M1 - 4, M2 - 30, M2bazo - 1, M2eo - 1, M3 - 4, M4 - 14, M4eo - 2, M5 - 3 , M6 - 4, M7 1 , blastic plasmacytoid dendritic cell neoplasm -2 .

In the study group, all patients underwent cytogenetic (G-banding) and molecular genetic (real-time PCR and direct automatic sequencing) analysis. Exons 1-4 of the NRAS gene were tested for molecular damage in accordance with the previously described sequencing methods [6-9]. In addition, 62 samples, including 17 - AML with normal karyotype and 18 - with unspecified, were examined for the presence of NPM1 gene mutations by molecular genetic and immunohistochemical methods [7].

Samples of peripheral blood and bone marrow aspirates of patients were examined. Peripheral blood was taken into the study only at a blastemia level of at least $2000 / \mu 1$. To prevent fragmentation and degradation of RNA, biosamples immediately after collection into vacuum tubes with EDTA and an anticoagulant were mixed with RNAlater TM Stabilization Solution RNA stabilization reagent and stored for up to 72 hours at $+4{ }^{\circ} \mathrm{C}$. Isolation of total RNA followed by reverse transcription into cDNA was carried out using M-MLV revertase and hexanucleotide primers with a random sequence. Isolated cDNA was amplified by PCR. DNA sequencing was performed on an automatic genetic analyzer in the direct and reverse sequences $[8,9]$. The sequencing results were processed using the MEGA X program [10]. The statistical hypothesis was tested using Fisher's exact test. 


\section{Results}

According to the results of a cytogenetic study, in 18 cases $(25.7 \%$, with $95 \%$ CI from 16.9 to $37.0 \%$ ), normal karyotype was detected, in 34 (48.6\%, with $95 \%$ CI from 37.2 to $60.0 \%$ ) - aberrant tumor karyotype. Among aberrant karyotypes, pseudodiploidy prevailed $(\mathrm{n}=18$, $25.7 \%$, with $95 \%$ CI from 16.9 to $37.0 \%$ ), incl. - specific anomalies associated with a favorable prognosis - 10 observations $(14.3 \%$, at $95 \%$ CI from 7.9 to $24.3 \%$; $\mathrm{t}(8 ; 21)$ (q22; q22) - 1, inv (16) ( p13; q22) - 6, t (15; 17) (q22; q21) - 3), with unfavorable - $3(4.3 \%$, at $95 \%$ CI from 1.5 to $11.9 \%$; $(11 ; 19)$ (q23; p13) - 1, del (5q) - 1, t (3; 3) (q21; q26) - 1), others - 5 (7.1\%, at $95 \%$ CI from 3.1 to $15.7 \%$; i (7) - 2, add (2) (q37) - 1, add (4) (p16) - 1 , ins $(7 ; 2)(\mathrm{q} 11 ; \mathrm{p} 23-\mathrm{p} 25)$ - 1). Aneuploidy and complex abnormalities of the karyotype were determined in $4(5.7 \%$, with $95 \%$ CI from 2.2 to $13.8 \%)$ and $11(15.7 \%$, with $95 \%$ CI from 9.0 to $26.0 \%$ ) cases, respectively. An additional immunohistochemical and molecular genetic study on NPM1 gene mutations made it possible to identify them in 8 samples with diploidy (44.4\%, with $95 \%$ CI from 24.6 to $66.3 \%$ ). Thus, according to the results of immunohistochemical and genetic studies, a favorable prognosis of AML was determined in 18 cases $(25.7 \%$, at $95 \%$ CI from 16.9 to $37.0 \%)$, the intermediate - in $15(21.4 \%$, at $95 \%$ CI from 13.4 to $32.4 \%$ ), unfavorable - 18 cases (25.7\%, with $95 \%$ CI from 16.9 to $37.0 \%$ ). In 19 samples $(27.1 \%$, at $95 \%$ CI from 18.1 to $38.5 \%)$, genetic lesions was not detected by immunohistochemistry, molecular genetics, and standard cytogenetic, respectively, and the prognosis of overall survival of these patients was not stratified [11].

In the exons 1-4 of the NRAS gene by direct sequencing, predictive implications of mutations were determined in 8 cases $(11.4 \%$, with $95 \%$ CI from 5.9 to $21.0 \%$ ). In all cases, they were represented by missense mutations in the coding sequence of exons 1-3, respectively, the involvement of the region blocking the internal GTPase activity of the Nras protein and stabilizing it in the active state was noted. This mechanism led to suppression of myeloid cell apoptosis and their uncontrolled proliferation. The karyotype of NRAS-positive leukemia was characterized by a wide spectrum of chromosomal abnormalities: in two cases, complex aberrations of the karyotype were determined (del (5)(q13) in combination with $\operatorname{del}(15)(\mathrm{q} 21) ; \mathrm{t}(3 ; 12)(\mathrm{q} 25 ; \mathrm{p} 13)$ in combined with dup(1)), in one case $-\mathrm{t}(8 ; 21)(\mathrm{q} 22 ; \mathrm{q} 22), \mathrm{t}(3 ; 3)(\mathrm{q} 21 ; \mathrm{q} 26), \mathrm{i}(7)$, diploidy and trisomy of the chromosome 13. In the remaining two samples, the type of the genetic abnormity was not specified. Accordingly, after an additional study of the mutational status of the NRAS gene by sequencing, the prognosis in all cases NRAS-positive AML was stratified as unfavorable. Morphologically, variants of AML with identified NRAS mutations were characterized as M0 (n = 3), M2 (n = 3), M2baso $(n=1)$ and M7 $(n=1)$. Clinically, these cases were characterized by primary resistance $(n=3)$, continuously relapse tumor progression $(n=2)$, or early mortality $(n=3)$. The average age of NRAS-positive patients was $51.0 \pm 7.3$ years (all patients were of employable age), the average follow-up was $8.4 \pm 8.2$ months.

Among the identified prognostically significant missense mutations, non-synonymous transversions predominated ( 5 cases, $7.1 \%$, with $95 \%$ CI from 3.1 to $15.7 \%$ ), one of which (T17A) was determined in one sample $(1.4 \%$, at $95 \% \mathrm{CI}$ from 0.3 to $7.7 \%)$, and two more (C181A, A182C) in two (2.9\% each, at 95\% CI from 0.8 to 9.8\%). Transitions were determined in three samples $(4.3 \%$, at $95 \% \mathrm{CI}$ from 1.5 to $11.9 \%)$, the first of which (G35A) was detected in two samples $(2.9 \%$, at $95 \%$ CI from 0.8 up to $9.8 \%$ ), in the second (G38A) - in one $(1.4 \%$, at $95 \% \mathrm{CI}$ from 0.3 to $7.7 \%)$. That is, in all cases the transitions of purine nucleotides were determined. All detected missense mutations were determined as isolated abnormality, were localized in exons $1-3$, their frequency was in the range 1.4$2.9 \%$.

Along with missense mutations, in one sample (1.4\%, with $95 \%$ CI from 0.3 to $7.7 \%$ ), the synonymous G360A transition was determined by the third position of the purine 
nucleotide in the codon, respectively, which did not lead to a replacement of the encoded amino acid due to degeneracy of the genetic code. In this case, the AML variant corresponded to M0 with an unspecified karyotype [11]. The wild-type NRAS coding sequence corresponding to the reference (NM_002524, GeneBank) one was detected in 61 cases $(87.1 \%$, with $95 \%$ CI from 77.3 to $93.1 \%$ ).

\section{Discussion}

According to international databases [2-4], the structure of NRAS point mutations in AML is dominated by non-synonymous substitutions of one nucleotide for another, leading to a change in the encoded amino acid, that is, a missense mutation. Their frequency is $96.0 \%$, among them, in most cases, guanine is replaced by adenine (55.2\%), and cytosine by adenine and adenine by guanine are replaced in $10.7 \%$ of cases each. In isolated cases, there are synonymous substituons and frame-shift insertions. The results obtained in our study generally corresponded to the above structure: missense mutations accounted for $88.9 \%$ of observations, frameshift deletions and insertions were not determined, synonymous substitutions - $11.1 \%$. Mutation of splicing sites in our study was not determined, because sequencing was limited to coding sequences of exons 1-4 of the NRAS gene.

In 39.2\%, changes are detected in codon 12 of exon 2 NRAS (G35A transition), aspartic acid instead of glycine is determined in the protein, respectively. A similar substitution with the formation of aspartic acid is observed in $21.9 \%$ of cases in codon 13 of exon 2 (G38A). The above mutations are recognized as oncogenic, because cause a violation of GTP hydrolysis and an increase in the amount of active protein N-ras-GTP, which, through a cascade of reactions, stimulates the neoplastic transformation of myeloid cells [12]. The mutation C181A (exon 3) found in two samples leads to the amino acid replacement of glutamine with lysine at position 13 of the polypeptide. Like previous mutations, it is oncogenic and is determined, according to the registers, in $13.7 \%$ of cases [12].

The case of the non-synonymous substitutions of T17A located in exon 1 revealed in our research is not described in international studies. In this regard, additional analysis of the impact of this mutation on the pathogenesis of AML is needed.

According to the synthetic lethal hypothesis, the expression of certain oncogenes is significantly activated in NRAS-positive malignancies compared to NRAS-negative tumors and normal tissues [13-14]. Among them, with NRAS-positive AML, the genes PTPN11, KRAS, CBL, and NF1 [4], which are involved in the constitutive activation of GTPase, are considered, resulting in a stable signal of proliferation inside the cell. On the contrary, the expression of some other genes in NRAS-mutated tumors is suppressed; accordingly, in our research, immunohistochemical and molecular genetic methods did not reveal mutations and changes in the intracellular expression of the NPM1 gene in NRAS-positive AML. This confirms the previously obtained data on the absence of a relationship between the indicated molecular events in AML oncogenesis $[8,9,15]$.

Thus, a comparative analysis of the results obtained in the study and international databases confirms that the identified NRAS mutations have an unfavorable prognostic value in AML. Moreover, missense mutations in exons 1-3 were determined in most samples, which corresponded to their most typical localization in the NRAS gene [4-5].

\section{Conclusion}

The clinical research of the NRAS gene mutations using direct automatic sequencing was significant for stratifying the prognosis of the overall survival of AML patients. In total, 
$11.4 \%$ of samples with prognostically unfavorable missense mutations were identified in the studied sample. Clinically, cases of AML with NRAS mutations were characterized by primary resistance to standard polychemotherapy, early mortality, or a continuously relapse course, the average follow-up was $8.4 \pm 8.2$ months. The average age of patients with NRAS missense mutations corresponded to the average values for the sample as a whole $51.0 \pm 7.3$ years, that is, mutations were determined mainly in patients of employable age. In general, the age range for detecting NRAS-positive AML was from 37 to 62 years, the median age for detecting mutations was 51.5 years.

It is also noteworthy that in the studied sample there were no mutations or changes in the intracellular localization of NPM1 gene expression in NRAS-positive AML, which indicates the absence of a relationship between these molecular events in AML oncogenesis. In general, the spectrum of the NRAS gene missense mutations identified in the study is consistent with that described in international databases for adult AML.

\section{References}

1. T. Herold, M. Rothenberg-Thurley, V.V. Grunwald, H. Janke, D. Goerlich, M.C. Sauerland, N.P. Konstandin, A. Dufour, S. Schneider, M. Neusser, B. Ksienzyk, P.A. Greif, M. Subklewe, A. Faldum, S.K. Bohlander, J. Braess, B. Wörmann, U. Krug, W.E. Berdel, W. Hiddemann, K. Spiekermann, K.H Metzeler, Leukemia (2020) doi 10.1038/s41375-020-0806-0

2. K. Marcus, C. Mattos, Clin. Cancer. Res., 21(8), 1810-1818 (2015) doi: 10.1158/1078-0432.CCR-14-2148

3. M.S. Miller, L.D. Miller, Frontiers in Genetics, 2, 100 (2012) doi:10.3389/fgene.2011.00100

4. C.M. Niemeye, Hematology Am. Soc. Hematol. Educ. Program., 2018(1), 307-312 (2018) doi:10.1182/asheducation-2018.1.307

5. J.A. Parker, C. Mattos, Cold Spring Harb. Perspect. Med., 8(8), pii: a031427 (2018) doi:10.1101/cshperspect.a031427

6. A.V. Vinogradov, A.V. Rezaykin, D.R. Salakhov, S.E. Ioschenko, A.G. Sergeev, Journal of Ural Medical Academic Science, 4, 124-127 (2013)

7. A.V. Vinogradov, Russian Journal of Oncology, 4, 34-35 (2013)

8. A.V. Vinogradov, A.V. Rezaykin, S.V. Sazonov, A.G. Sergeev, Genes and Cells 13(3), 70-74 (2018) doi:10.23868/201811036

9. A.V. Vinogradov, A.V. Rezaikin, A.G. Sergeev, Journal of Bashkir State University, 19(3), 845-847 (2014)

10. S. Kumar, G. Stecher, M. Li, C. Knyaz, K. Tamura, Mol. Biol. Evol., 35(6), 15471549 (2018) doi:10.1093/molbev/msy096

11. A.V. Vinogradov, A.V. Rezaykin, D.V. Izotov, A.G. Sergeev, Journal of Ural Medical Academic Science, 4, 38-51 (2016) doi:10.22138/2500-0918-2016-14-4-38-51

12. G. Buhrman, G. Wink, C. Mattos, Structure, 15(12), 1618-1629 (2007) doi:10.1016/j.str.2007.10.011

13. J. Downward, Clin. Cancer Res., 21(8), 1802-1809 (2015) doi:10.1158/10780432.CCR-14-2180

14. A.C. Kimmelman, Clin. Cancer. Res., 21(8), 1828-1834 (2015) doi:10.1158/10780432.CCR-14-2425

15. A.V. Vinogradov, A.V. Rezaykin, S.V. Sazonov, A.G. Sergeev, M.Yu. Kapitonova, Genes and Cells, 14(4), 19-24 (2019) doi: 10.23868/201912027 